\title{
circ_0003170 aggravates human hippocampal neuron injuries by regulating the miR-421/CCL2 axis in cells models of epilepsy
}

\author{
Fang Chen, Hongjia Zheng, Wenyu Zhang, Jie Kang, Qiao Liu, Juan Pu and Ling Yang \\ Department of Neurology, Panzhihua Municipal Central Hospital, Panzhihua, China
}

\begin{abstract}
Emerging evidence proposes that circular RNAs (circRNAs) are involved in epileptogenesis. This study aimed to investigate the role and the function mechanism of circ_0003170 in epilepsy models in vitro. Epilepsy models were established in human hippocampal neurons treated by magnesium-free $\left(\mathrm{Mg}^{2+}\right.$-free) solution. The expression of circ_003170, miR-421 and C-C motif chemokine ligand 2 (CCL2) was detected by qRT-PCR. The putative interaction between miR-421 and circ_003170 or CCL2 was validated by dual-luciferase reporter assay and RIP assay. The protein level of CCL2 was detected by Western blot. Cell viability was detected by CCK-8 assay, and cell cycle and apoptosis were monitored by flow cytometry. The content of superoxide dismutase (SOD) and malondialdehyde (MDA) and the activity of caspase- 3 were assessed using commercial kits. The results showed that circ_0003170 and CCL2 expression was enhanced, while miR-421 expression was declined in temporal lobe epilepsy serum specimens and $\mathrm{Mg}^{2+}$-free-induced neurons. circ_0003170 knockdown ameliorated $\mathrm{Mg}^{2+}$-free-induced cell cycle arrest, oxidative stress and apoptosis in neurons by enriching miR-421. Further analysis presented that miR-421 overexpression alleviated $\mathrm{Mg}^{2+}$-freeinduced cell injuries by depleting CCL2. CCL2 overexpression reversed the effects of circ_0003170 knockdown. Overall, circ_0003170 knockdown ameliorated $\mathrm{Mg}^{2+}$-free-induced human hippocampal neuron injuries by mediating the miR-421/CCL2 axis.
\end{abstract}

Key words: circ_0003170 - miR-421 - CCL2 - Epilepsy - Hippocampal neuron - $\mathrm{Mg}^{2+}$-free

\section{Introduction}

Epilepsy is a chronic neurological disease that affects more than 50 million people worldwide, characterized by recurrent and unexplained attacks (Unehan et al. 2011). Epilepsy is thought to be caused by an abnormal hypersynchronous electrical activity of the neuronal network caused by the imbalance of excitatory and inhibitory neurons in the central nervous system (CNS) (Unterrainer et al. 2004; Guilhoto 2017). Typical clinical features of epilepsy are recurrent seizure disorders and widespread or localized muscle cramps and convulsions (Wan and Yang 2020).

Electronic supplementary material. The online version of this article (doi: 10.4149/gpb_2020045) contains Supplementary Material.

Correspondence to: Fang Chen, Department of Neurology, Panzhihua Municipal Central Hospital, No. 34 Yikang Street, East District, Panzhihua, 617000, China

E-mail: tmlapp@163.com
Epileptogenesis is still a challenge to overcome, with molecular, cellular, and structural dynamic changes (Pitkanen et al. 2015). Although accumulating studies have explored the mechanism of epilepsy, the candidate pathogenic factors and mechanisms of epilepsy are still lacking. Exploring the molecular mechanism of genetic regulation in epilepsy helps explain the development of epilepsy.

Non-coding RNAs (ncRNAs), including long ncRNAs (lncRNAs), microRNAs (miRNAs) and circular RNAs (circRNAs), regulate the expression of numerous target genes by several regulatory mechanisms (Jimenez-Mateos and Henshall 2013; Lee et al. 2018). circRNA harbors a covalently closed circular structure and is produced by "back-splicing" by which downstream exon is reversely spliced to upstream exon (Memczak et al. 2013). Expression profiling reveals specific changes to brain circRNA levels in temporal neocortex tissues of temporal lobe epilepsy (TLE) patients (Li et al. 2018), hinting that circRNAs may participate in epilepsy progression. Hippocampus neural network contains a large number of neurons, and its dys- 
function or injury plays a crucial role in TLE (Gong et al. 2014). Hippocampal neurons treated by magnesium-free $\left(\mathrm{Mg}^{2+}\right.$-free) solution were frequently used as TLE in vitro models (Gong et al. 2014), which provided available models to explore the function of ncRNAs (Geng et al. 2018). Although the vital role of circRNAs in epilepsy is recognized, few studies have been conducted on circRNAs in vivo or in vitro models of epilepsy. Here, we focused on circ_0003170 (derived from STK24), which was highly expressed in TLE specimens analyzed by circRNA profiles Li et al. (2018). However, its function and potential functional mechanism in epilepsy are unknown.

It is well known that partial circRNAs target specific miRNAs through miRNA response elements to play competing endogenous RNA (ceRNA) effects (Zhang et al. 2018). Numerous miRNAs were also reported to be implicated in epilepsy pathogenesis, including miR-421. Nevertheless, the role of miR-421 in $\mathrm{Mg}^{2+}$-free-induced hippocampal neurons is deficient, and the associated mechanism of miR-421 linked to circ_0003170 is not unclosed. Moreover, miRNAs modulate gene expression mainly via sequence-specific binding sites within the 3' untranslated region (3'UTR) of mRNA transcripts (Jimenez-Mateos and Hendshall 2013). C-C motif chemokine ligand 2 (CCL2) was reported to trigger epileptic seizure by mediating inflammatory responses (Bozzi and Caleo 2016). CCL2 is a target of miR-421, which was verified in this study. However, the interaction between miR-421 and CCL2 is not previously explored, and further mechanism of CCL2 in epilepsy associated with circ_0003170 is worth clarifying.

In the current research, epilepsy in vitro models were generated using human hippocampal neurons with the treatment of $\mathrm{Mg}^{2+}$-free. The expression of circ_0003170 was monitored in TLE serum specimens and $\mathrm{Mg}^{2+}$-freeinduced neurons, and the function of circ_0003170 on cell viability, cycle, apoptosis and oxidative stress was investigated in vitro. In addition, the circ_0003170/miR421/CCL2 network was assembled to reveal a potential regulatory mechanism of circ_0003170, aiming to reveal the novel pathogenesis of epilepsy through circRNA regulatory network.

\section{Materials and Methods}

\section{Serum specimens}

A total of 30 TLE patients and 10 normal volunteers were recruited from Panzhihua Municipal Central Hospital and participated in the study. Blood samples from TLE patients and normal subjects were utilized to collect serum specimens by centrifugation. All serum specimens exposed to liquid nitrogen were stored at $-80^{\circ} \mathrm{C}$ conditions. Each participant had signed written informed consent and approved the use of serum specimens. This study was implemented with the authorization of Panzhihua Municipal Central Hospital.

\section{Experimental cells}

Human hippocampal neurons were purchased from Sciencell (catalog: \#1540; Carlsbad, CA, USA) and cultured in the recommended medium (Neuronal Medium; catalog: \#1521; Sciencell). Human hippocampal neurons were cultured at $37^{\circ} \mathrm{C}$ conditions with $5 \% \mathrm{CO}_{2}$. After plating for 3 days, the culture medium was replaced every 3 days. The neurons at days 7-21 were used in vitro for the following assay.

To induce epileptiform cell models, hippocampal neurons cultured in vitro at day 6 were exposed to magnesium-free $\left(\mathrm{Mg}^{2+}\right.$-free) solution containing $\mathrm{NaCl}(145 \mathrm{mM}), \mathrm{KCl}(2.5$ $\mathrm{mM}), \operatorname{HEPES}(10 \mathrm{mM}), \mathrm{CaCl}_{2}(2 \mathrm{mM})$, glucose $(10 \mathrm{mM})$ and glycine (0.002 $\mathrm{mM}$ ) for continuing $3 \mathrm{~h}$ (Jiang et al. 2010; Dai et al. 2019). Then, treated neurons were re-cultured in Neuronal Medium in standard conditions. Untreated hippocampal neurons for normal culture were used as the control.

\section{Quantitative real-time polymerase chain reaction ( $q R T-P C R$ )}

Firstly, total RNA was extracted using RNAiso Plus Kit (Takara, Dalian, China) and used for cDNA synthesis using the Real Time One Step RT-PCR Kit (Takara) or using miRNA First Strand Synthesis Kit (Takara) following the protocols. cDNA was then used for qRT-PCR using a TB Green qRT-PCR Kit (Takara) under a CFX96 Touch PCR system (Bio-Rad, Hercules, CA, USA). $\beta$-actin or U6 was served as the internal control, and the method of $2^{-\Delta \Delta C t}$ was used to calculate relative expression. The primer sequences were listed as below: circ_0003170, F: 5'-GGAGTGGGGTTATTGGGTCA-3' and R: 5'- TGGCCAGGTAGTCAGACAAG-3'; miR-421, F: 5'-TGCGGATCAACAGACATTAATTGGGC-3' and R: 5'-CCAGTGCAGGGTCCGAGGT-3'; CCL2, F: 5'-AGCAGCAAGTGTCCCAAAGA-3' and R: 5'-TTTGCTTGTCCAGGTGGTCC-3'; $\beta$-actin, F: 5'- CCAAGGCCAACCGCGAGAAGATGAC-3' and R: 5'-AGGGTACATGGTGGTGCCGCCAGAC-3'; U6, F: 5'-GGAACGCTTCACGAATTTG-3' and R: 5'-ATTGGAACGATACAGAGAAGATT-3'

\section{Bioinformatics analysis}

Bioinformatics analysis was performed to predict the potential targets of circ_0003170 and miR-421. In brief, the target miRNAs of circ_0003170 were analyzed by Starbase (http://starbase.sysu.edu.cn/) and Circular RNA interactome 
(https://circinteractome.nia.nih.gov/). The target mRNAs of miR-421 was analyzed by Starbase.

\section{Dual-luciferase reporter assay}

The targeting sites between miR-421 and circ_0003170 or CCL2 3'UTR were mutated to form mutant sequences of circ_0003170 or CCL2. Then the wild- and mutant-type of circ_0003170 sequence and CCL2 sequence were cloned into pmirGLO reporter plasmid (Promega, Madison, WI, USA), named as circ_0003170-WT, circ_0003170-MUT, CCL2WT and CCL2-MUT. Human hippocampal neurons were transfected with miR-421 or miR-NC and circ_0003170WT, circ_0003170-MUT, CCL2-WT or CCL2-MUT using Lipofectamine 3000 (Invitrogen, Carlsbad, CA, USA). After maintaining for $48 \mathrm{~h}$, the luciferase activity in cells was checked using the Dual-Luciferase Assay System (Promega).

\section{RNA immunoprecipitation (RIP) assay}

Magna RIP Kit (Millipore, Bedford, MA, USA) was utilized to conduct RIP assay to validate the interaction between miR-421 and circ_0003170 or CCL2 in line with the directions. Briefly, human hippocampal neurons were lysed, and cell lysate was mixed with RIP buffer with magnetic beads conjugated with Argonaute 2 antibody (anti-Ago2) or Immunoglobulin G antibody (anti-IgG; control). Afterwards, the RNA samples bound to beats were isolated, purified and used for qRT-PCR.

\section{Cell transfection}

For functional analysis, circ_0003170 knockdown or overexpression was accomplished using small interference RNA (siRNA) (si-circ_0003170\#1, \#2 and \#3) or pcDNA overexpression vector (circ_0003170) (Genepharma, Shanghai, China), with siRNA random sequence or pcDNA vector as a negative control (si-NC or pcDNA). miR-421 mimic (miR-421) or miR-421 inhibitor (anti-miR-421) was used for miR-421 overexpression or inhibition (Ribobio, Guangzhou, China), with mimic negative control (miR-NC) or inhibitor negative control (anti-miR-NC) as control. CCL2 overexpression was conducted using pcDNA overexpression vector (CCL2) (Genepharma), also with pcDNA vector as control. According to the protocol's procedures, cell transfection was carried out using Lipofectamine 3000 (Invitrogen, Carlsbad, CA, USA).

\section{RNase R treatment}

Total RNA was isolated and exposed to $2 \mathrm{U} / \mu \mathrm{g}$ RNase $\mathrm{R}$ (Epicentre, Madison, WI, USA) for $15 \mathrm{~min}$ at $37^{\circ} \mathrm{C}$. Then, qRT-PCR was performed to check the expression of circ_0003170 and its linear mRNA STK24. The primers used of STK24 were: F, 5'-CCCGGCATGCAGAACCTAAA-3' and R, 5'-CCAAAGGAGCCCTTCCCAAT-3'.

\section{Subcellular distribution}

Nuclear RNA and cytoplasmic RNA were isolated using the Cytoplasmic \& Nuclear RNA purification kit (Norgen Biotek, Thorold, Canada) according to the guidelines. The isolated RNA was used for qRT-PCR analysis to detect the abundance of circ_0003170 in subcellular parts. GAPDH or U6 was used as the control in cytoplasm and nucleus, respectively.

\section{Cell counting kit-8 (CCK-8) assay}

Cells with transfection were plated into 96-well plates at a density of 5000 cells/well. Cells were then incubated for $48 \mathrm{~h}$, and $10 \mu \mathrm{lCCK}-8$ reagent was added into each well for another $2 \mathrm{~h}$. The absorbance at $450 \mathrm{~nm}$ was monitored using a microplate reader (Bio-Rad) to observe cell viability.

\section{Flow cytometry assay}

For cell cycle analysis, after transfection, cells were collected, following by trypsinization, and resuspended in phosphatebuffered saline (PBS). Cells were next fixed in 70\% ethanol at $4^{\circ} \mathrm{C}$ overnight and collected for staining using propidium iodide (PI) staining solution from a Cell cycle analysis kit (Beyotime, Shanghai, China) for $30 \mathrm{~min}$ at $37^{\circ} \mathrm{C}$ in the dark, followed by detection on a flow cytometer (BD Biosciences, San Jose, CA, USA).

For apoptosis analysis, cells were collected and resuspended in PBS. Then, cells were exposed to binding buffer and orderly stained with Annexin V-fluorescein isothiocyanate (FITC) and PI from a Cell apoptosis detection kit (Beyotime) according to the protocol, for $15 \mathrm{~min}$ at room temperature in the dark. The apoptotic cells were analyzed using a flow cytometer (BD Biosciences).

\section{Detection of superoxide dismutase (SOD) and malondialdehyde (MDA)}

The levels of SOD and MDA were detected using Total SOD Assay Kit (Beyotime) and Lipid Peroxidation MDA Assay Kit (Beyotime), respectively. All experimental procedures were conducted in line with the protocol.

\section{Caspase-3 activity}

The activity of caspase- 3 was measured using a GreenNuc Caspase-3 Assay Kit (Beyotime) referring to the manufacturer's protocol. The result was monitored to detect optical density (OD) of caspase- 3 activity at $405 \mathrm{~nm}$. 


\section{Western blot}

The expression of CCL 2 at the protein level was detected by Western blot. In brief, total protein was extracted, quantified and separated by electrophoresis. The isolated protein was transferred into polyvinylidene fluoride (PVDF) membranes followed by blockage. The membranes were then exposed to the primary antibody against CCL2 (anti-CCL2; sc-32771; Santa Cruz Biotechnology, Santa Cruz, CA, USA) or $\beta$-actin (anti- $\beta$-actin; sc-47778; Santa Cruz Biotechnology) at $4^{\circ} \mathrm{C}$ overnight and subsequently exposed to the secondary antibody (sc-516102; Santa Cruz Biotechnology) at $37^{\circ} \mathrm{C}$ for $1.5 \mathrm{~h}$. The protein plots were finally examined using an enhanced chemiluminescence kit (Beyotime). The original gels containing protein signals are shown in Figure S1 (see Supplementary material).

\section{Statistical analysis}

Data from at least three independent experiments were statistically analyzed using GraphPad Prism 5.0 (GraphPad Software, La Jolla, CA, USA) and finally shown as the mean \pm standard deviation (SD). In addition, differences between two groups or among multiple groups were analyzed by
Student's $t$-test or analyses of variance with Tukey post hoc test. The correlation of expression levels between two groups was analyzed by Spearman's correlation test. $p$ value $\leq 0.05$ was regarded to be statistically significant.

\section{Results}

The expression of circ_0003170 was elevated, while the expression of miR-421 was declined in serum specimens from TLE patients and $\mathrm{Mg}^{2+}$-free-induced human hippocampal neurons

QRT-PCR analysis was performed to ensure the expression of circ_0003170 and miR-421 in TLE serum specimens and cell models. Firstly, the formation of circ_0003170 was validated by RT-qPCR, and we found that circ_0003170 could be amplified by divergent primers from cDNA but not gDNA. In contrast, linear transcripts, including GAPDH and STK23 (parental mRNA of circ_0003170), could be amplified by convergent primers from both $\mathrm{cDNA}$ and gDNA (Fig. 1A). As shown in Figure 1B and C, the expression of circ_0003170 was significantly increased in serum
A

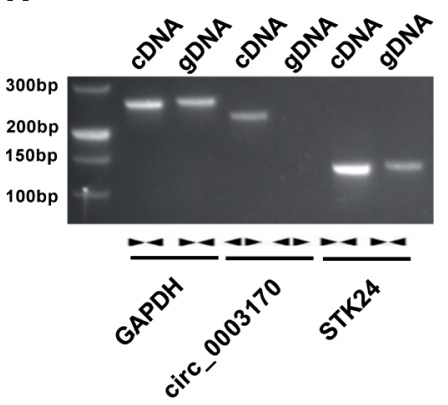

D

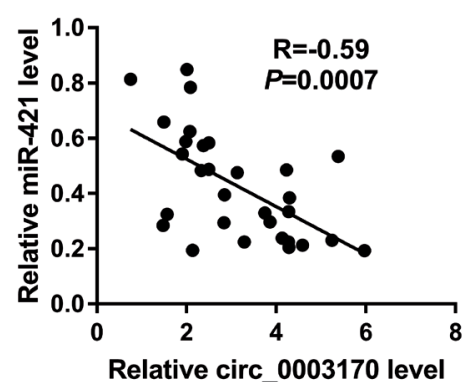

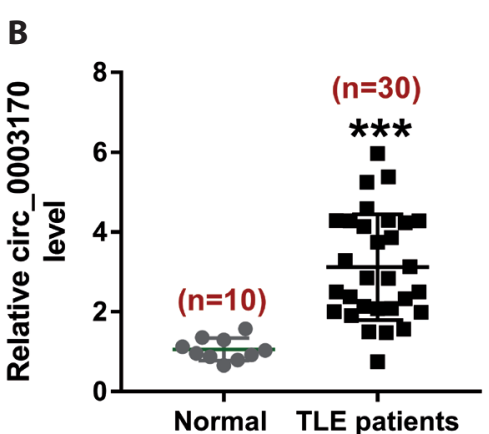

E

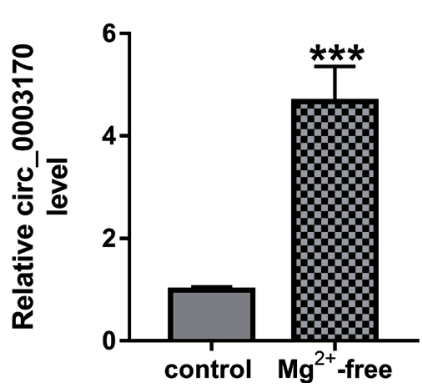

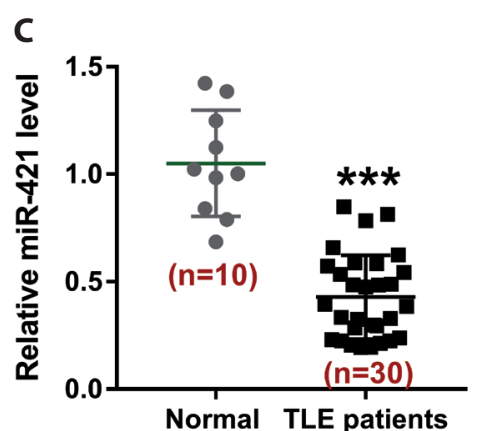

$\mathbf{F}$

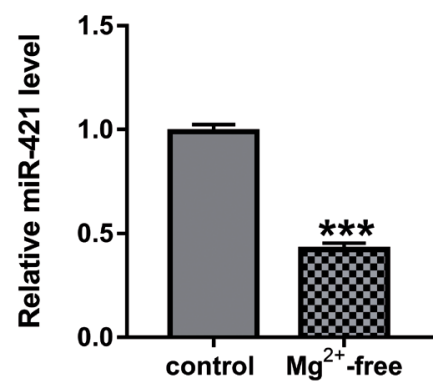

Figure 1. circ_0003170 was upregulated, while miR-421 was downregulated in temporal lobe epilepsy (TLE) serum specimens and $\mathrm{Mg}^{2+}$ free-induced human hippocampal neurons. A. The formation of circ_0003170 was identified by divergent primers, using GAPDH and STK24 as linear control. B, C. The expression of circ_0003170 and miR-421 in serum specimens from TLE patients and normal subjects was detected using qRT-PCR. D. Spearman's correlation analysis revealed the correlation between miR-421 and circ_0003170 expression in TLE serum specimens. E, F. The expression of circ_0003170 and miR-421 in $\mathrm{Mg}^{2+}$-free-induced human hippocampal neurons was detected using qRT-PCR. ${ }^{* * *} p<0.001$. 

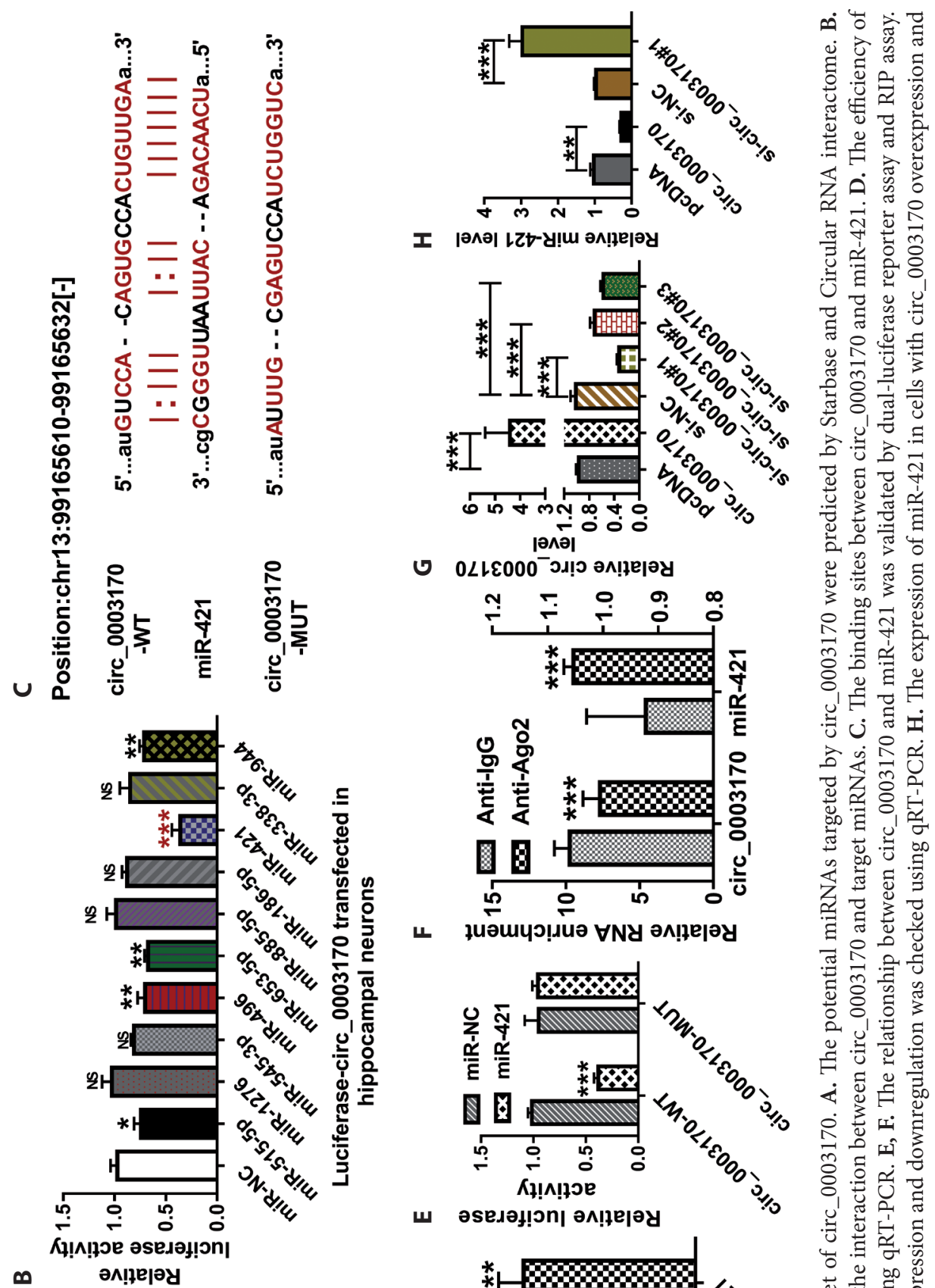

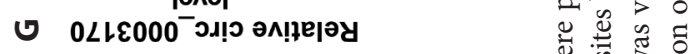

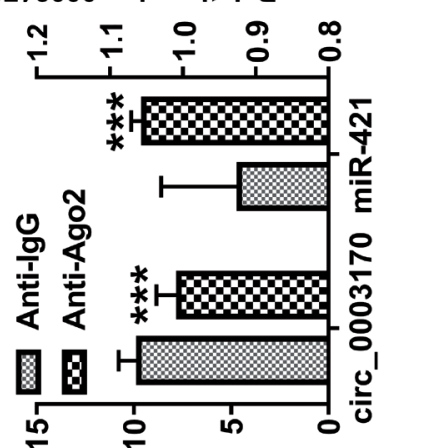

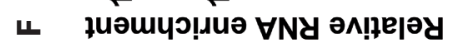

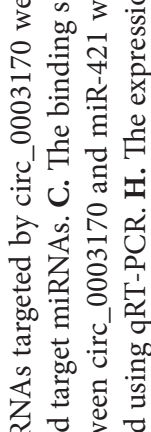

$\infty$

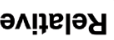
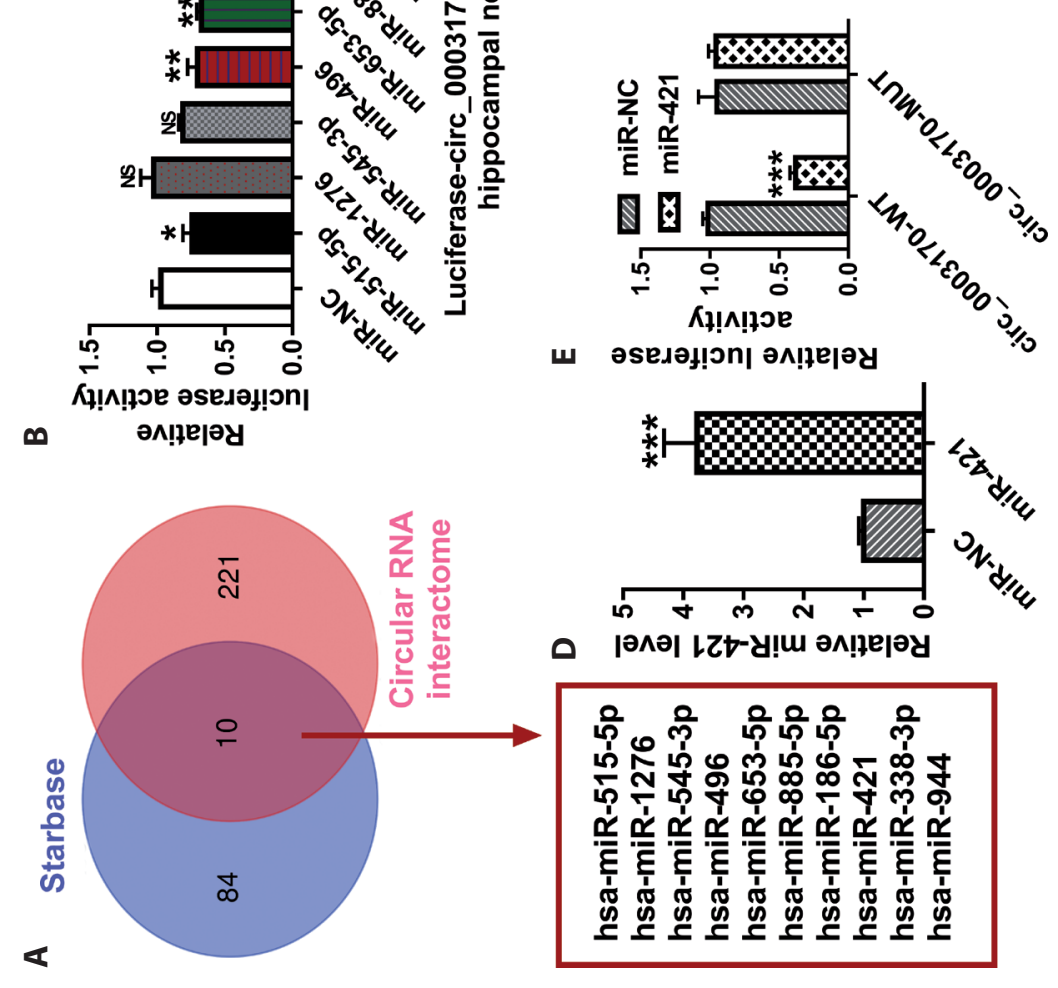

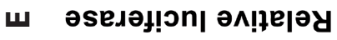

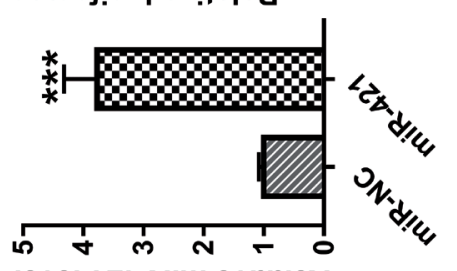

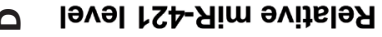
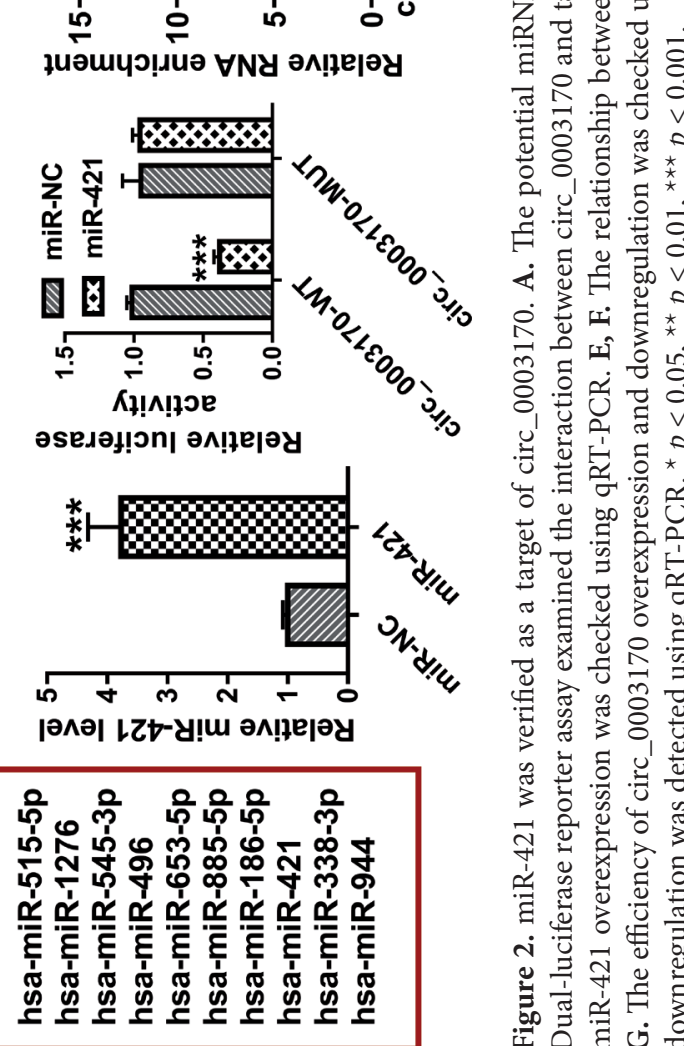


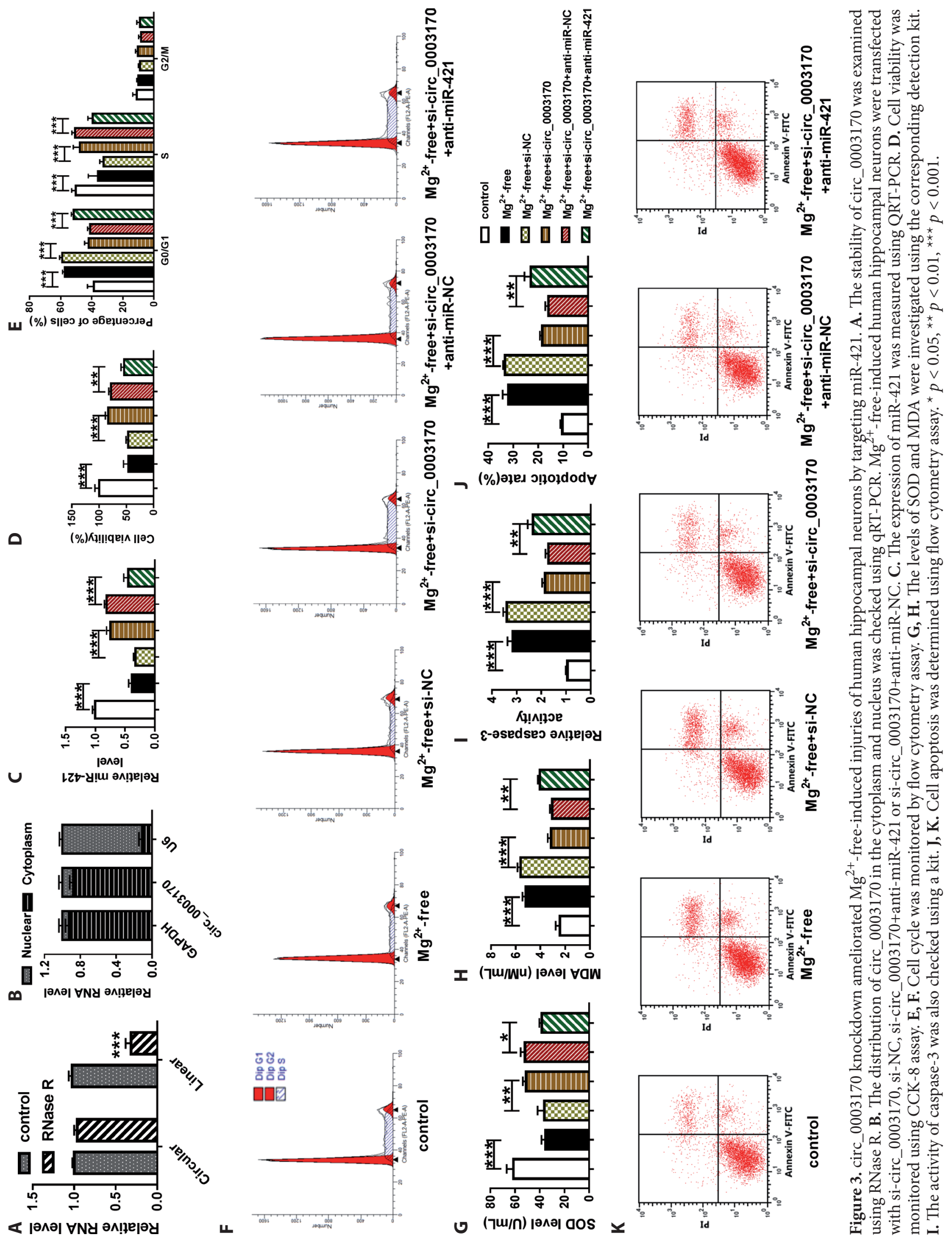


specimens from TLE patients compared with that from normal subjects, while the expression pattern of miR-421 was opposite to circ_0003170. Besides, the expression of miR-421 in TLE serum specimens was negatively correlated with the expression of circ_0003170 (Fig. 1D). As expected, circ_0003170 was notably upregulated in $\mathrm{Mg}^{2+}$-free-induced hippocampal neurons compared to control, while miR-421 was downregulated in $\mathrm{Mg}^{2+}$-free-induced hippocampal neurons (Fig. 1E,F). The dysregulation of circ_0003170 and miR-421 hinted that they might be involved in the development of TLE.

\section{miR-421 was identified to be a target of circ_0003170}

miR-421 as a target of circ_0003170 was predicted by both Starbase and Circular RNA interctome. Meanwhile, a total of ten miRNAs were simultaneously predicted by two bioinformatics tools (Fig. 2A). Ten miRNA mimics were cotransfected with circ_0003170luciferase reporter plasmid in human hippocampal neurons, respectively, and the results showed that miR-421 mimic could reduce luciferase activity more effectively (Fig. 2B). Hence, miR-421 was chosen for further analyses. Besides, the target relationship between miR-421 and circ_0003170 was validated by dual-luciferase reporter assay and RIP assay. As shown in Fig. 2C, mutanttype sequence of circ_0003170 was designed according to its wild-type sequence. miR-421 mimic transfection strikingly promoted the expression of miR-421 in human hippocampal neurons (Fig. 2D) and diminished the luciferase activity in human hippocampal neurons transfected with circ_0003170-WT but not circ_0003170-MUT (Fig. 2E). RIP assay displayed that circ_0003170 and miR-421 could be significantly enriched in the RIP group with Anti-Ago2 compared to Anti-IgG (Fig. 2F). For circ_0003170 overexpression and knockdown efficiency analysis, the data showed that circ_0003170 expression was remarkably enhanced in cells transfected with circ_0003170 compared to pcDNA, and circ_0003170 expression was remarkably declined in cells transfected with si-circ_0003170 (\#1, \#2 and \#3), suggesting that overexpression efficiency and knockdown efficiency are available (Fig. 2G). Moreover, the expression of miR-421 was reduced with circ_0003170 overexpression and strengthened with circ_0003170 knockdown (Fig. 2H). Collectively, miR421 was a target of circ_0003170 and negatively regulated by circ_0003170.

\section{circ_0003170 knockdown ameliorated $\mathrm{Mg}^{2+}$-free-induced injuries of human hippocampal neurons by regulating miR-421}

circ_0003170 was stably expressed in human hippocampal neurons due to its significant resistance to the digestion of RNase $\mathrm{R}$ relative to corresponding linear mRNA (Fig. 3A).
Subcellular distribution analysis showed that the abundance of circ_0003170 in the cytoplasm was significantly higher than that in the nucleus (Fig. 3B). For functional experiments, partial human hippocampal neurons treated with $\mathrm{Mg}^{2+}$-free were transfected with si-circ_0003170 or si-NC, and partial human hippocampal neurons treated with $\mathrm{Mg}^{2+}$-free were cotransfected with si-circ_0003170+antimiR-421 or si-circ_0003170+anti-miR-NC. The expression of miR-421 inhibited in $\mathrm{Mg}^{2+}$-free-induced cells was largely recovered in cells with the transfection of si-circ_0003170 but partly reduced in cells with the cotransfection of circ_0003170+anti-miR-421 (Fig. 3C). CCK-8 assay showed lower cell viability in $\mathrm{Mg}^{2+}$-free-induced human hippocampal neurons, and the transfection of si-circ_0003170 notably recovered cell viability, which was lessened by the cotransfection of circ_0003170+anti-miR-421 (Fig. 3D). Flow cytometry cycle assay exhibited that $\mathrm{Mg}^{2+}$-freeinduced cell cycle arrest was relieved by the transfection of si-circ 0003170 but promoted by the cotransfection of circ_0003170+anti-miR-421 (Fig. 3E,F). Besides, the level of SOD was weakened by $\mathrm{Mg}^{2+}$-free, while the transfection of si-circ_0003170 improved the level of SOD, and the cotransfection of circ_0003170+anti-miR-421 impaired the level of SOD (Fig. 3G). The level of MDA was always opposite to the levels of SOD (Fig. 3H). Caspase-3 was an important indicator of apoptosis, and the activity of caspase- 3 was significantly elevated in $\mathrm{Mg}^{2+}$-free-induced human hippocampal neurons. However, circ_0003170 knockdown diminished caspase-3 activity, while combined circ_0003170 knockdown and miR-421 inhibition restored the activity of caspase-3 (Fig. 3I). The result was verified by flow cytometry apoptosis assay that introduced that apoptotic rate enhanced by $\mathrm{Mg}^{2+}$-free was ameliorated by si-circ_0003170 transfection and partly recovered by circ_0003170+anti-miR-421 transfection (Fig. 3J,K). In short, circ_0003170 knockdown mediated miR-421 to ameliorate $\mathrm{Mg}^{2+}$-free-induced human hippocampal neuron injuries.

\section{miR-421 bound to CCL2 3'UTR, thus suppressing the expression of CCL2}

We further used Starbase to analyze the potential target mRNAs of miR-421. There were several binding sites between miR-421 and CCL2 3'UTR, and the sequence of CCL2 3'UTR mutated at the miR-421 binding sites was assembled to conduct dual-luciferase reporter assay (Fig. 4A). Luciferase activity of human hippocampal neurons with cotransfection of miR-421 and CCL2-WT was significantly declined, while cotransfection of miR-421 and CCL2-MUT never reduced the luciferase activity in neurons (Fig. 4B). RIP assay also verified the interaction between CCL2 and miR-421 (Fig. 4C). The data from qRT-PCR and Western blot showed an increase of CCL2 expression in TLE serum 
specimens and $\mathrm{Mg}^{2+}$-free-induced human hippocampal neurons (Fig. 4D,E). Besides, miR-421 expression was negatively correlated with CCL2 mRNA expression in TLE serum specimens (Fig. 4F). Moreover, miR-421 transfection strikingly promoted the expression of miR-421 but weakened the expression of CCL2, while anti-miR-421 transfection strikingly reduced the expression of $\mathrm{miR}-421$ but reinforced the expression of CCL2 (Fig. 4G,H). In conclusion, CCL2 was a target of miR-421, and the expression patterns of miR-421 and CCL2 were opposite in TLE.

miR-421 restoration ameliorated $\mathrm{Mg}^{2+}$-free-induced injuries of human hippocampal neurons by downregulating CCL2

To monitor the function of miR-421 and CCL2, partial $\mathrm{Mg}^{2+}$-free-induced human hippocampal neurons were transfected with miR-421, miR-NC, miR-421+CCL2 or miR$421+$ pcDNA. The expression of CCL2 was impaired in $\mathrm{Mg}^{2+}$ free-induced cells transfected with miR-421 but recovered in $\mathrm{Mg}^{2+}$-free-induced cells transfected with miR-421+CCL2 (Fig. 5A). miR-421 transfection promoted cell viability of human hippocampal neurons blocked by $\mathrm{Mg}^{2+}$-free relative to miR-NC, while miR-421+CCL2 cotransfection impaired cell viability relative to miR-421+pcDNA (Fig. 5B). Besides, miR421 overexpression lessened $\mathrm{Mg}^{2+}$-free-induced cell cycle arrest relative to $\mathrm{miR}-\mathrm{NC}$, and $\mathrm{miR}-421+\mathrm{CCL} 2$ transfection promoted cell cycle arrest relative to miR- $421+$ pcDNA transfection (Fig. 5C). miR-421+CCL2 cotransfection blocked the level of SOD, which was induced by miR-421 transfection alone (Fig. 5D), while miR-421+CCL2 cotransfection strengthened the level of MDA which was inhibited by miR-421 transfection alone in $\mathrm{Mg}^{2+}$-free-induced human hippocampal neurons (Fig. 5E). The activity of caspase-3 was suppressed by miR-421 transfection but recovered by miR$421+$ CCL2 cotransfection in $\mathrm{Mg}^{2+}$-free-induced human hippocampal neurons (Fig. 5F). The apoptotic rate was also repressed in $\mathrm{Mg}^{2+}$-free-induced cells transfected with miR421 but improved in cells transfected with miR-421+CCL2 (Fig. 5G). All data suggested that miR-421 restoration lessened $\mathrm{Mg}^{2+}$-free-induced injuries, which was reversed by CCL2 overexpression.

\section{circ_0003170 sponged miR-421 to upregulate CCL2}

The expression of circ_0003170 was positively correlated with CCL2 mRNA expression in TLE serum specimens
A

\begin{tabular}{|c|c|}
\hline \multicolumn{2}{|c|}{ Position:chr17:32583939-32583946 of CCL2 3'UTR } \\
\hline CCL2-WT & 5'...auaaugAAUU-UUGUUUGUUGAu...3' \\
\hline & \|\|$|\|:\|| \mid \|$ \\
\hline $\mathrm{miR}-421$ & $3^{\prime} . . . c g c g g g$ UUAUU UCAGACA ACUa....5' \\
\hline 2 & 5'...auaaugGGCC-UUGGUGUCCAGu. \\
\hline
\end{tabular}

B

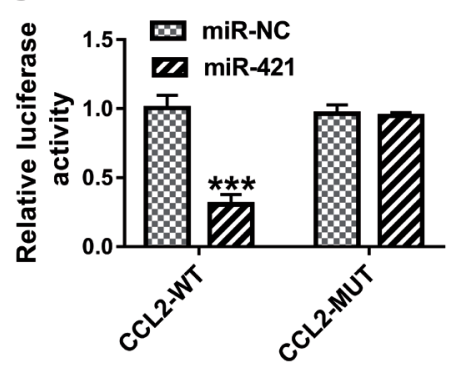

D

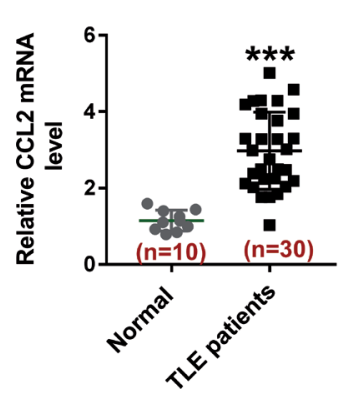

E

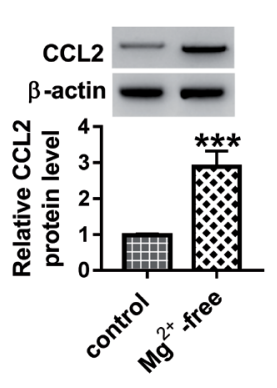

F

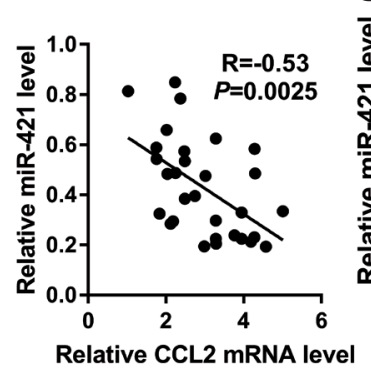

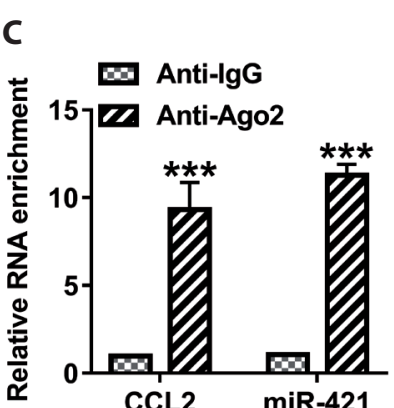

H

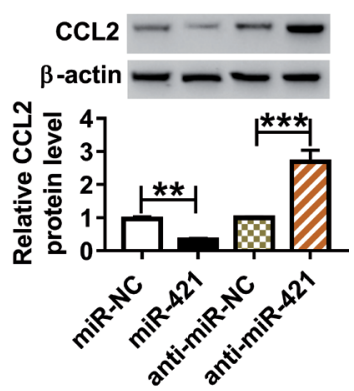

Figure 4. CCL2 was a target of miR-421. A. The binding sites between miR-421 and CCL2 3'UTR were analyzed by Starbase. B, C. The interaction between miR-421 and CCL2 was verified by dual-luciferase reporter assay and RIP assay. D. The expression of CCL2 in serum specimens from temporal lobe epilepsy (TLE) patients or normal subjects was measured using qRT-PCR. E. The expression of CCL2 in $\mathrm{Mg}^{2+}$-free-induced human hippocampal neurons was measured using Western blot. $\mathrm{F}$. The correlation between miR-421 expression and CCL2 expression in TLE serum was analyzed using Spearman's correlation analysis. G. The efficiency of miR-421 overexpression or inhibition was examined using qRT-PCR. H. The expression of CCL2 in cells with miR-421 overexpression or inhibition was detected using Western blot. ${ }^{* *} p<0.01,{ }^{* * *} p<0.001$. 
A

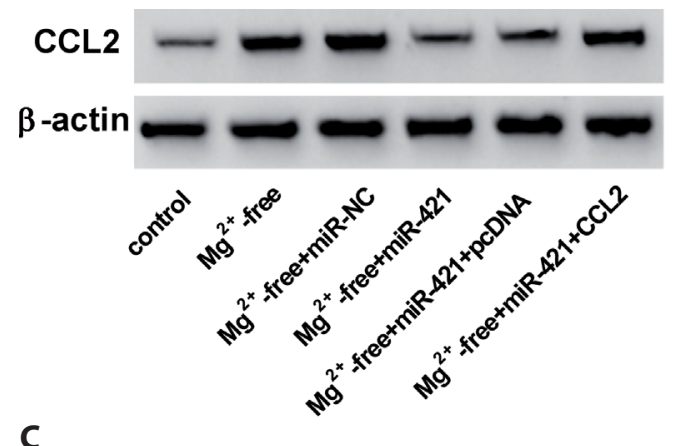

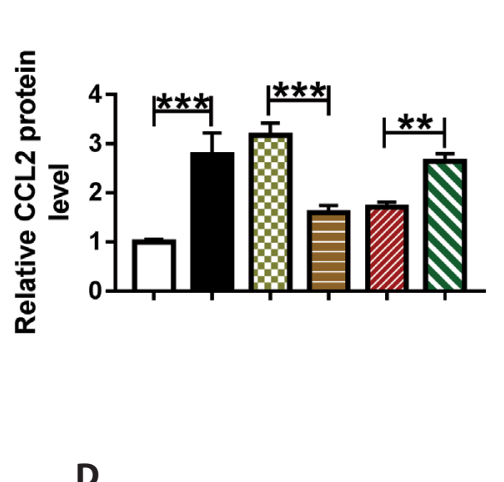

B

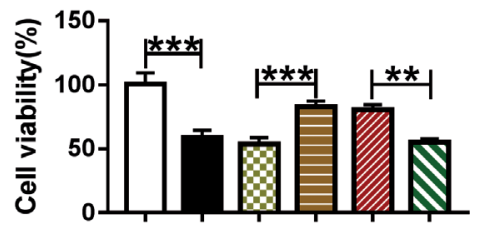

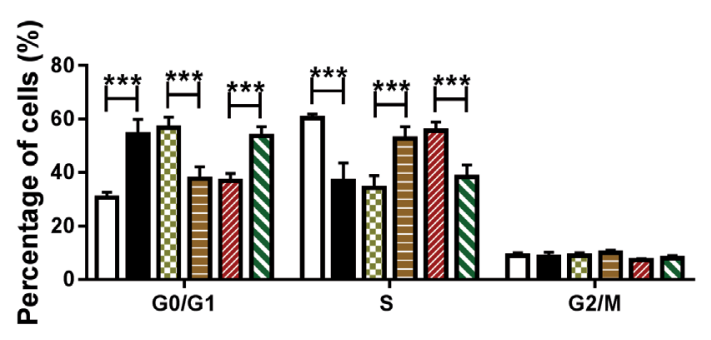

$\mathbf{E}$

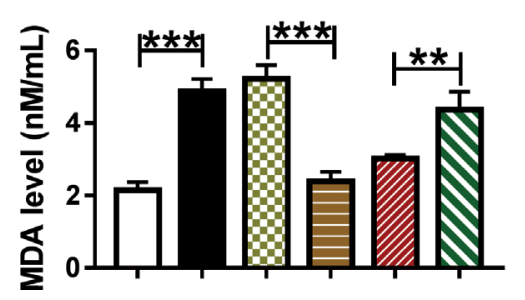

$\mathbf{F}$

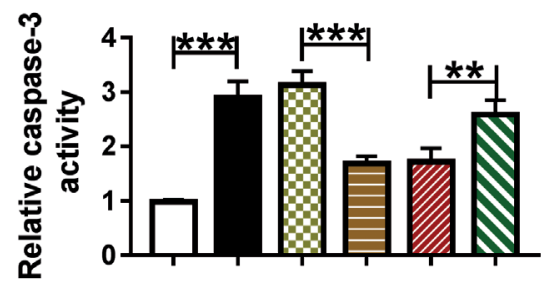

G

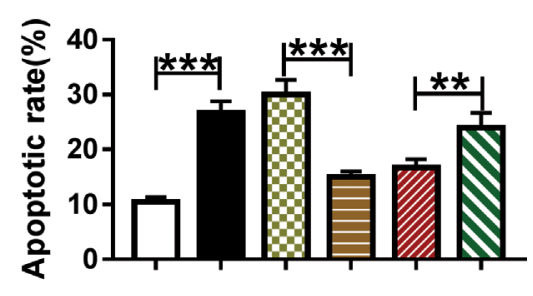

Figure 5. miR-421 restoration ameliorated $\mathrm{Mg}^{2+}$-free-induced injuries of human hippocampal neurons by degrading CCL2. $\mathrm{Mg}^{2+}$-freeinduced human hippocampal neurons were transfected with miR-421, miR-NC, miR-421+CCL2 or miR-421+pcDNA. A. The expression of CCL2 in these cells was detected by Western blot. B. Cell viability was assessed using CCK-8 assay. C. Cell cycle was monitored by flow cytometry assay. D-F. The levels of SOD and MDA and the activity of caspase-3 were checked using the corresponding kits. G. Cell apoptosis was checked using flow cytometry assay. ${ }^{* *} p<0.01,{ }^{* * *} p<0.001$.

(Fig. 6A). Moreover, the protein level of CCL2 was also significantly promoted in human hippocampal neurons transfected with circ_0003170 but decreased in cells transfected with circ_0003170+miR-421 (Fig. 6B). On the contrary, the expression level of CCL2 was lessened in cells transfected with si-circ_0003170 but recovered in cells transfected with si-circ_0003170+anti-miR-421 (Fig. 6C). The data implied that circ_0003170 regulated the expression of CCL2 by targeting miR-421.

\section{CCL2 overexpression rescued the effects of circ_0003170 knockdown}

$\mathrm{Mg}^{2+}$-free-induced human hippocampal neurons were transfected with si-circ_0003170 alone or si-circ_0003170+CCL2. The protein level of CCL2 was decreased in $\mathrm{Mg}^{2+}$-free- induced human hippocampal neurons transfected with si-circ_0003170 but restored in cells transfected with si-circ_0003170+CCL2 (Fig. 7A). Cotransfection of sicirc_0003170+CCL2 diminished cell viability, which was promoted by si-circ_0003170 transfection alone in $\mathrm{Mg}^{2+}$ free-induced human hippocampal neurons (Fig. 7B). Cotransfection of si-circ_0003170+CCL2 induced cell cycle arrest, which was lightened by si-circ_0003170 transfection alone (Fig. 7C). Besides, circ_0003170 knockdown-promoted SOD level and circ_0003170 knockdown-suppressed MDA level were overturned by combined CCL2 overexpression (Fig. 7D,E). In addition, caspase-3 activity and apoptotic rate were largely blocked in cells transfected with si-circ_0003170 while were heightened in cells cotransfected with si-circ_0003170+CCL2 (Fig. 7F,G). All data indicated that circ_0003170 knockdown-blocked human hippocampal 

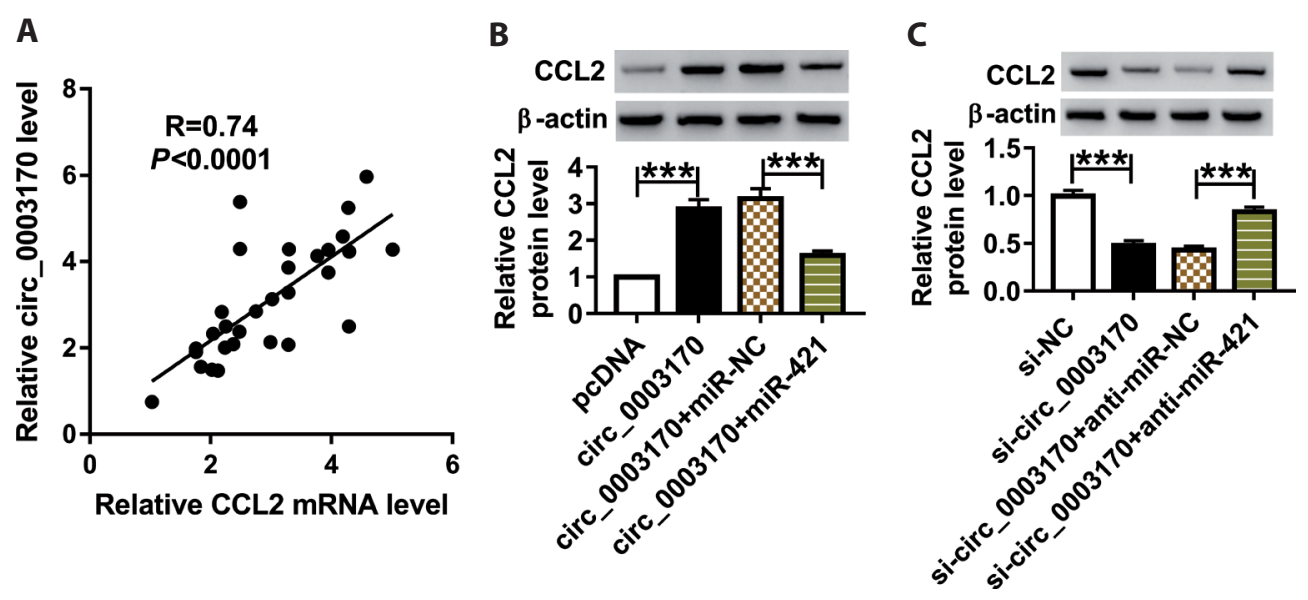

Figure 6. circ_0003170 activated the expression of CCL2 by targeting miR421. A. The correlation between circ_0003170 expression and CCL2 expression in TLE serum was ascertained by Spearman's correlation analysis. B. The expression of CCL2 in human hippocampal neurons transfected with circ_0003170, pcDNA, circ_0003170+miR-421 or circ_0003170+miR-NC was detected by Western blot. C. The expression of CCL2 in human hippocampal neurons transfected with si-circ_0003170, si-NC, si-circ_0003170+anti-miR-421 or si-circ_0003170+anti-miR-NC was detected by Western blot. ${ }^{* * *} p<0.001$.

A

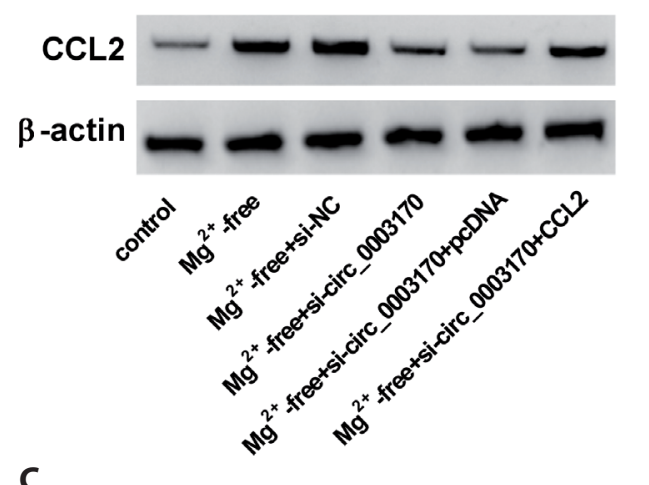

C

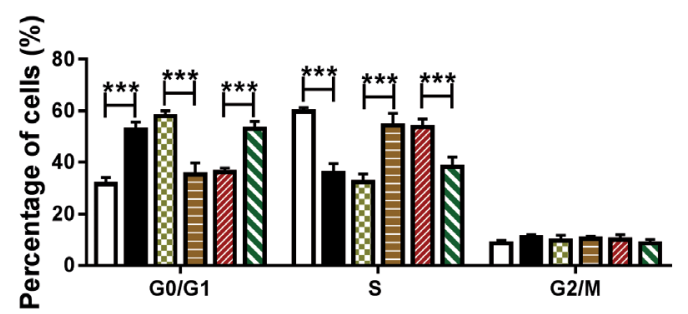

B

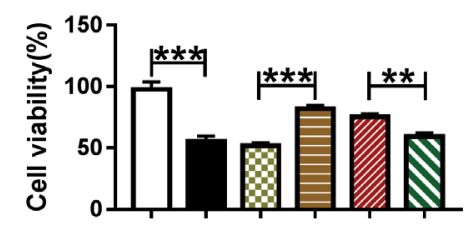

D

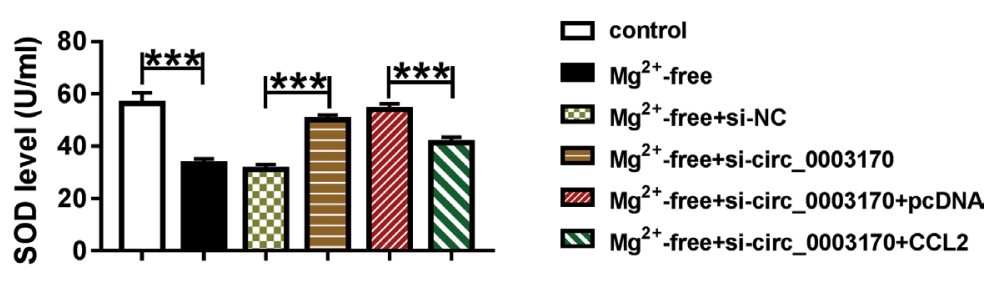

E

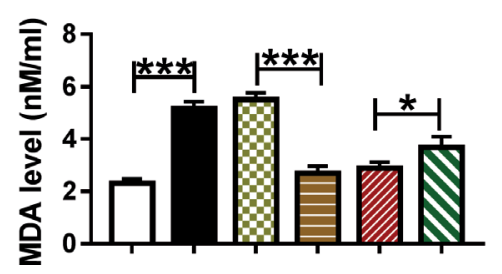

$\mathbf{F}$

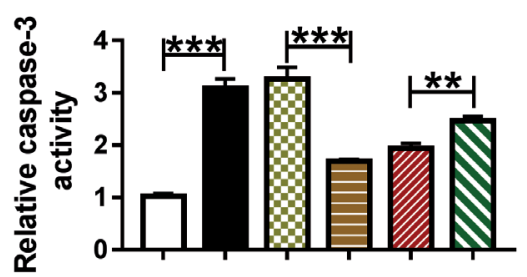

$\mathbf{G}$

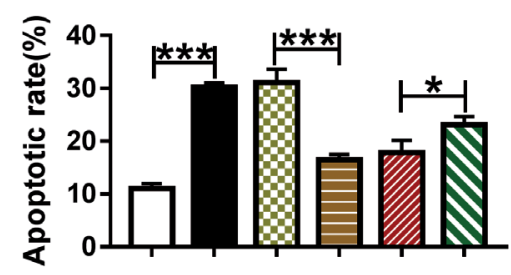

Figure 7. CCL2 overexpression rescued the effects of circ_0003170 knockdown in $\mathrm{Mg}^{2+}$-free-induced human hippocampal neurons. $\mathrm{Mg}^{2+}$-free-induced human hippocampal neurons were transfected with si-circ_0003170, si-NC, si-circ_0003170+CCL2 or sicirc_0003170+pcDNA. A. The expression of CCL2 was detected by Western blot. B. Cell viability was checked by CCK-8 assay. C. Cell cycle was monitored using flow cytometry assay. D-F. The levels of SOD and MDA and the activity of caspase-3 were determined by the corresponding kit. G. Cell apoptosis was evaluated by flow cytometry. ${ }^{\star} p<0.05,{ }^{\star *} p<0.01,{ }^{\star * *} p<0.001$. 
neuron injuries induced by $\mathrm{Mg}^{2+}$-free, including cell viability inhibition, cell cycle arrest, oxidative stress and apoptosis promotion, were improved by CCL2 overexpression.

\section{Discussion}

The properties of circRNAs make them promising biomarkers for CNS diseases. For example, the expression of circRNAs in CNS is abundant (Rybak-Wolf et al. 2015), and circRNAs are more stably expressed in CNS (Chen and Shuman 2016). Thus, identifying the expression alteration and functional role of circRNAs in CNS diseases, such as epilepsy, may provide novel diagnostic tools and therapeutic targets. Regrettably, circRNAs are poorly investigated in epilepsy. A previous study introduced that circ_0067835 was strikingly downregulated in plasma and tissues of TLE patients, and overexpression of circ_0067835 suppressed refractory epilepsy progression via activating the expression of FOXO3a by targeting miR-155 (Gong et al. 2018). Besides, high-throughput sequencing technology screened numerous differently expressed circRNAs in TLE specimens, which provided several epilepsy-specific circRNAs (Gong et al. 2018; Li et al. 2018). Here, to further understand the pathomechanism of epilepsy, we functionally investigated a poorly studied circRNA, circ_0003170, which was identified to be abundantly expressed in TLE samples (Li et al. 2018). Consistent with the data from the previous study, our results showed that circ_0003170 was aberrantly upregulated in TLE serum specimens and cell models. Yuan et al. utilized $\mathrm{Mg}^{2+}$-free-induced epilepsy cell model to investigate the function of miR-132 on epileptogenesis (Yuan et al. 2016), and Geng et al. (2018) also used $\mathrm{Mg}^{2+}$-free physiological solution to induce epileptiform activity and explored the function of lncRNA UCA1 in this cell model. Similarly, in $\mathrm{Mg}^{2+}$-free-induced human hippocampal neurons, we noted that knockdown of circ_0003170 ameliorated $\mathrm{Mg}^{2+}$ free-induced cell cycle arrest, cell apoptosis and oxidative stress of human hippocampal neurons, thus protecting the normal functions of hippocampal neurons. Mechanically, we validated that miR-421 was a target of circ_0003170.

Previous research explored the role of miR-421 in epilepsy mice model and unclosed that miR-421 expression was weakened in mice model, and miR-421 enrichment blocked apoptosis and autophagy and promoted proliferation of mouse hippocampal neurons (Wen et al. 2018). In line with the data, our results discovered that the expression of miR421 was prominently declined in TLE serum specimens and $\mathrm{Mg}^{2+}$-free-induced hippocampal neurons. In function, miR-421 restoration relieved $\mathrm{Mg}^{2+}$-free-induced neuron cell cycle arrest, cell apoptosis and oxidative stress, and miR-421 inhibition counteracted the effects of circ_0003170 knockdown. These data suggested that miR-421 prevented the progression of epilepsy.
Further mechanism analysis disclosed that miR-421 directly bound to CCL2. CCL2 was acknowledged as a proepileptogenic factor (Korotkov et al. 2020), and numerous studies documented that CCL2 enhanced epileptic effects by mediating partial or systemic inflammation (Cerri et al. 2016). Besides, CCL2 was also reported to be a target of miR206, and enforced expression of CCL2 abolished the effects of miR-206 and recovered epilepsy-induced brain injuries $(\mathrm{Wu}$ et al. 2019). Here, we reported that CCL2 was a target of miR421 , and the abundance of CCL2 was aberrantly enhanced in TLE serum specimens and $\mathrm{Mg}^{2+}$-free-induced neurons. Functionally, CCL2 overexpression restored $\mathrm{Mg}^{2+}$-freeinduced neuron cell cycle arrest, cell apoptosis and oxidative stress, which were impaired by miR-421 enrichment. These functions of CCL2 were accordant with the previous findings. More importantly, circ_0003170 could indirectly regulate the expression of CCL2 by targeting miR-421. Functional analysis noticed that the blocked cell cycle arrest, cell apoptosis and oxidative stress caused by circ_0003170 knockdown were rescued by CCL 2 overexpression, supporting the view that CCL2 was a pro-epileptogenic factor.

\section{Conclusion}

Taken together, the present study monitored that the expression of circ_0003170 and CCL2 was increased while the expression of miR-421 was decreased in TLE serum specimens and $\mathrm{Mg}^{2+}$-free-induced human hippocampal neurons. circ_0003170 triggered neuron injuries, including cell cycle arrest, apoptosis and oxidative stress, partly by regulating the miR-421/CCL2 axis. Our data supported that circ_0003170 participated in the development of epilepsy and provided new vision into the molecular mechanism of epilepsy progression.

Conflict of interest. The authors declare that they have no financial conflicts of interest.

\section{References}

Bozzi Y, Caleo M (2016): Epilepsy, seizures, and inflammation: Role of the C-C motif ligand 2 chemokine. DNA Cell Biol. 35, 257-260

https://doi.org/10.1089/dna.2016.3345

Cerri C, Genovesi S, Allegra M, Pistillo F, Puntener U, Guglielmotti A, Perry VH, Bozzi Y, Caleo M (2019): The chemokine CCL2 mediates the seizure-enhancing effects of systemic inflammation. J. Neurosci. 36, 3777-3788 https://doi.org/10.1523/JNEUROSCI.0451-15.2016

Chen W, Schuman E (2016): Circular RNAs in brain and other tissues: a functional enigma. Trends Neurosci. 39, 597-604 https://doi.org/10.1016/j.tins.2016.06.006 
Dai H, Wang P, Mao H, Mao X, Tan S, Chen Z (2019): Dynorphin activation of kappa opioid receptor protects against epilepsy and seizure-induced brain injury via PI3K/Akt/Nrf2/HO-1 pathway. Cell Cycle 18, 226-237 https://doi.org/10.1080/15384101.2018.1562286

Geng JF, Liu X, Zhao HB, Fan WF, Geng JJ, Liu XZ (2018): LncRNA UCA1 inhibits epilepsy and seizure-induced brain injury by regulating miR-495/Nrf2-ARE signal pathway. Int. J. Biochem. Cell Biol. 99, 133-139 https://doi.org/10.1016/j.biocel.2018.03.021

Gong GH, An FM, Wang Y, Bian M, Wang D, Wei CX (2018): Comprehensive circular RNA profiling reveals the regulatory role of the circRNA-0067835/miR-155 pathway in temporal lobe epilepsy. Cell. Physiol. Biochem. 51, 1399-1409 https://doi.org/10.1159/000495589

Gong XW, Li JB, Lu QC, Liang PJ, Zhang PM (2014): Effective connectivity of hippocampal neural network and its alteration in Mg2+-free epilepsy model. PloS One 9, e92961 https://doi.org/10.1371/journal.pone.0092961

Guilhoto LM (2017): Absence epilepsy: Continuum of clinical presentation and epigenetics? Seizure 44, 53-57 https://doi.org/10.1016/j.seizure.2016.11.031

Jiang Q, Wu Y, Wang J, Wu X, Qin J, Jiang Y (2010): Characterization of developing rat cortical neurons after epileptiform discharges. Int. J. Dev. Neurosci. 28, 455-463 https://doi.org/10.1016/j.ijdevneu.2010.06.006

Jimenez-Mateos EM, Henshall DC (2013): Epilepsy and microRNA. Neuroscience 238, 218-229 https://doi.org/10.1016/j.neuroscience.2013.02.027

Korotkov A, Broekaart DWM, Banchaewa L, Pustjens B, Van Scheppingen J, Anink JJ, Baayen JC, Idema S, Gorter JA, van Vliet EA, Aronica E (2020): microRNA-132 is overexpressed in glia in temporal lobe epilepsy and reduces the expression of proepileptogenic factors in human cultured astrocytes. Glia 68, 60-75 https://doi.org/10.1002/glia.23700

Lee WJ, Moon J, Jeon D, Kim TJ, Yoo JS, Park DK, Lee ST, Jung KH, Park LI, Jung KY, et al. (2018): Possible epigenetic regulatory effect of dysregulated circular RNAs in epilepsy. PloS One 13, e0209829 https://doi.org/10.1371/journal.pone.0209829

Li J, Lin H, Sun Z, Kong G, Yan X, Wang Y, Wang X, Wen Y, Liu X, Zheng H, et al. (2018): High-throughput data of circular RNA profiles in human temporal cortex tissue reveals novel insights into temporal lobe epilepsy. Cell. Pysiol. Biochem. 45, 677-691 https://doi.org/10.1159/000487161

Linehan C, Tellez-Zenteno JF, Burneo JG, Berg AT (2011): Future directions for epidemiology in epilepsy. Epilepsy Behav. 22, 112-117 https://doi.org/10.1016/j.yebeh.2011.06.006

Memczak S, Jens M, Elefsinioti A, Torti F, Krueger J, Rybak A, Maier L, Mackowiak SD, Gregersen LH, Munschauer M, et al. (2013): Circular RNAs are a large class of animal RNAs with regulatory potency. Nature 495, 333-338 https://doi.org/10.1038/nature11928

Pitkanen A, Lukasiuk K, Dudek FE, Staley KJ (2015): Epileptogenesis. Cold Spring Harb. Perspect. Med. 5, a022822 https://doi.org/10.1101/cshperspect.a022822

Rybak-Wolf A, Stottmeister C, Glazar P, Jens M, Pino N, Giusti S, Hanan M, Behm M, Bartok O, Ashwall-Fluss R (2015): Circular RNAs in the mammalian brain are highly abundant, conserved, and dynamically expressed. Mol. Cell 58, 870-885 https://doi.org/10.1016/j.molcel.2015.03.027

Unterrainer JM, Rahm B, Kaller CP, Leonhart R, Quiske K, HoppeSeiler K, Meier C, Müller C, Halsband U (2004): Planning abilities and the Tower of London: is this task measuring a discrete cognitive function? J. Clin. Exp. Neuropsychol. 26, 846-856 https://doi.org/10.1080/13803390490509574

Wan Y, Yang ZQ (2020): Lncrna Neatl affects inflammatory response by targeting miR-129-5p and regulating Notch signaling pathway in epilepsy. Cell Cycle 19, 419-431 https://doi.org/10.1080/15384101.2020.1711578

Wen X, Han Xr, Wang Yj, Wang S, Shen M, Zhang ZF, Fan SH, Shan Q, Wang L, Li MQ (2018): MicroRNA-421 suppresses the apoptosis and autophagy of hippocampal neurons in epilepsy mice model by inhibition of the TLR/MYD88 pathway. J. Cell. Physiol. 233, 7022-7034 https://doi.org/10.1002/jcp.26498

Wu Z, Liu Y, Huang J, Huang Y, Fan L (2019): MiR-206 inhibits epilepsy and seizure-induced brain injury by targeting CCL2. Cytotechnology 71, 809-818 https://doi.org/10.1007/s10616-019-00324-3

Yuan J, Huang H, Zhou X, Liu X, Ou S, Xu T, Li R, Ma L, Chen Y (2016): MicroRNA-132 Interact with p250GAP/Cdc42 pathway in the hippocampal neuronal culture model of acquired epilepsy and associated with epileptogenesis process. Neural Plast. 2016, 5108489 https://doi.org/10.1155/2016/5108489

Zhang Y, Zhang H, An M, Zhao B, Ding H, Zhang Z, He Y, Shang $\mathrm{H}, \mathrm{Han} X$ (2018): Crosstalk in competing endogenous RNA networks reveals new circular RNAs involved in the pathogenesis of early HIV infection. J. Transl. Med. 16, 332 https://doi.org/10.1186/s12967-018-1706-1

Received: August 20, 2020

Final version accepted: December 16, 2020 
doi: $10.4149 / g p b \_2020045$

Supplementary Material circ_0003170 aggravates human hippocampal neuron injuries by regulating the miR-421/CCL2 axis in cells models of epilepsy

Fang Chen, Hongjia Zheng, Wenyu Zhang, Jie Kang, Qiao Liu, Juan Pu and Ling Yang Department of Neurology, Panzhihua Municipal Central Hospital, Panzhihua, China

Supplementary Figures

Figure 1A

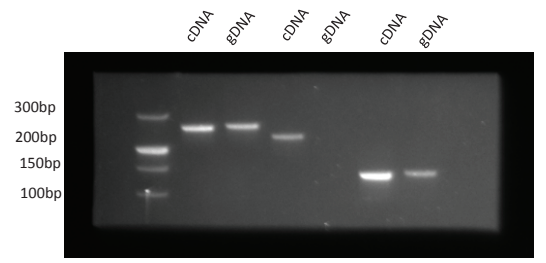

GAPDH circ_0003170 STK24

Figure 4H

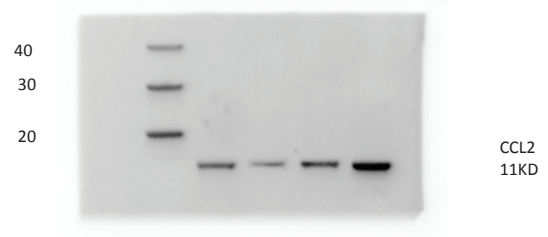

Figure 5A

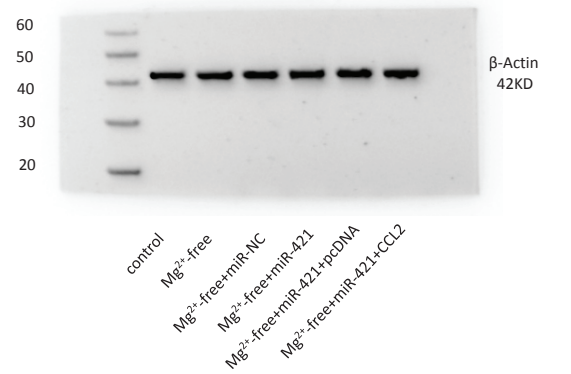

Figure 4E

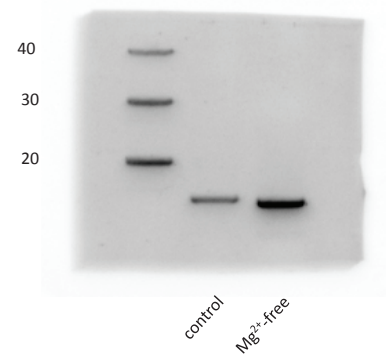

Figure 4H

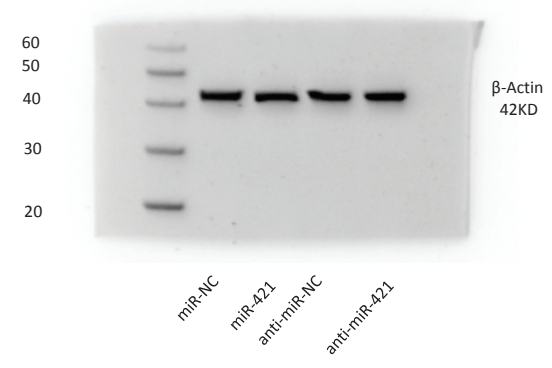

Figure 6B
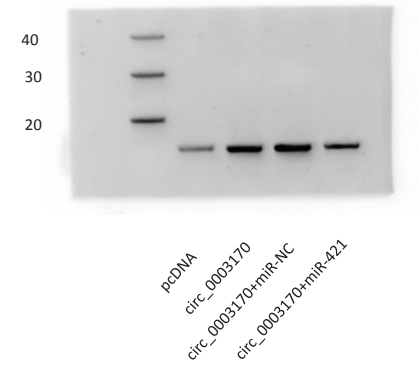

Figure 4E

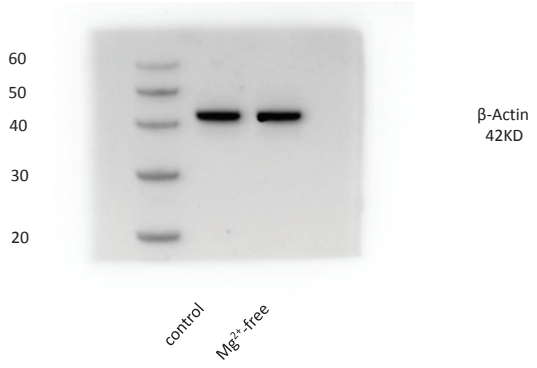

Figure 5A

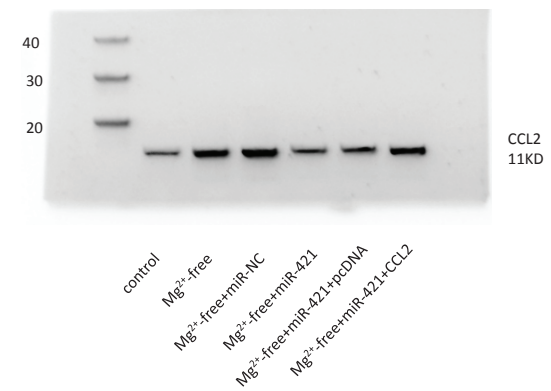

Figure 6B

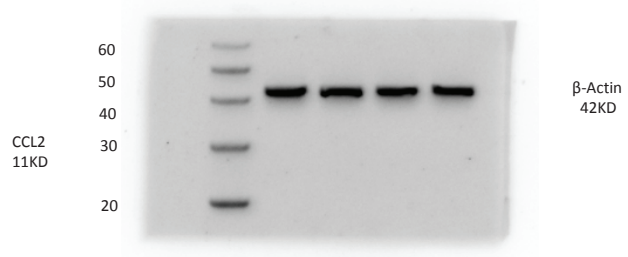


Figure 6C

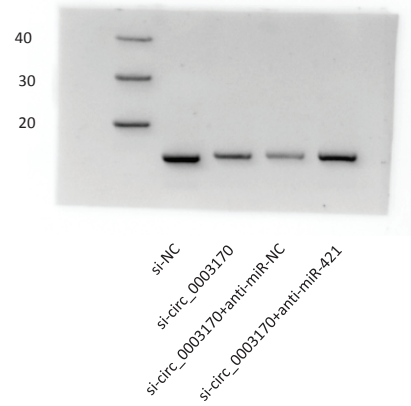

Figure 7A

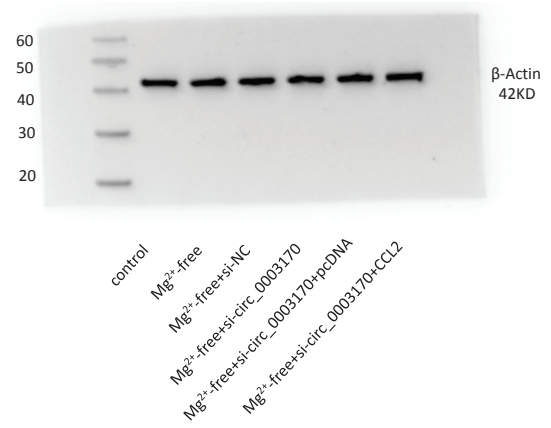

\section{Figure 6C}

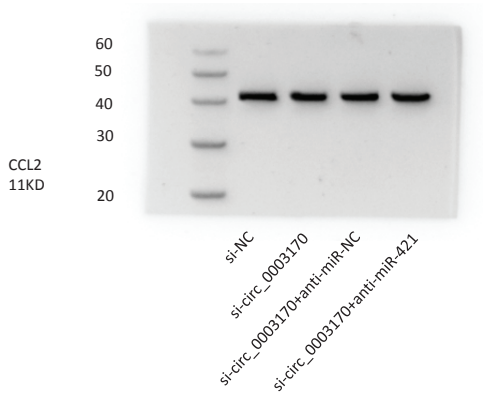

Figure 7A

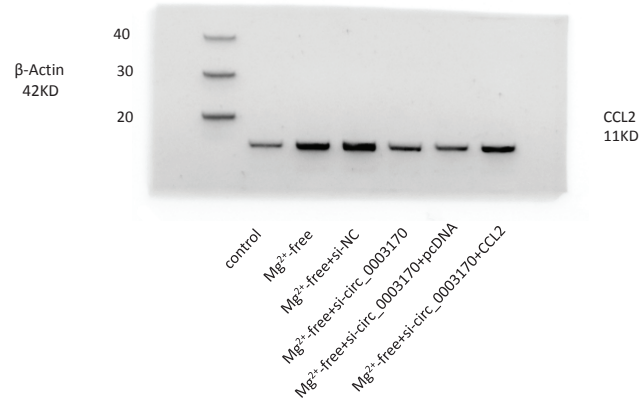

Figure S1. Original Western blots of investigated proteins (blots corresponds to the numbering of figures in the main text). 